\title{
MATERIAL DOCENTE EN FORMATO ELECTRÓNICO PARA LA ENSEÑANZA DE LA ASIGNATURA DE Procedimientos QUirúrgicos en El Grado EN MEdicina
}

\section{TEACHING MATERIALS IN ELECTRONIC FORMAT FOR MEDICAL EDUCATION IN THE SUBJECT OF SURGICAL PROCEEDINGS IN THE DEgREE OF MEDICINE}

\author{
Pedro López Cillero, Sebastián Rufián Peña, \\ Paula Moreno Casado, Antonio Álvarez Kindelán, \\ Antonio Luque Molina \\ PLOPEZC@YAHOO.ES
}

Received: 25/05/2017 Accepted: 20/02/2018

\begin{abstract}
Resumen
La Cirugía es la parte de la Medicina que tiene por objeto curar las enfermedades por medio de operaciones hechas con las manos o a través de instrumentos. Hasta ahora, la enseñanza de la asignatura de "Procedimientos Quirúrgicos", que corresponde a los estudiantes de segundo curso del Grado en Medicina de la Universidad de Córdoba, se ha llevado a cabo según el estilo tradicional. Se ha llevado a cabo un proyecto específico de elaboración de una herramienta multimedia, en concreto, un ebook, con el contenido teórico y práctico de la asignatura de Procedimientos Quirúrgicos, para mejorar la docencia médica según las directrices del Espacio Europeo de Educación Superior (EEES), fomentando así el desarrollo de una educación médica de calidad.
\end{abstract}

Palabras clave: ebook, educación médica, cirugía, material docente.

\begin{abstract}
Surgery is the branch of Medicine concerned with diseases and conditions requiring or amenable to operative or manual procedures. To date, teaching the subject of Surgical Procedures for 2nd-year students of the degree of Medicine has been done in a traditional manner. We have developed a specific project, with the creation of a multimedia resource consisting in an ebook with the theoretical and practical content of the Surgical Proceedings subject, in order to improve medical learning following the principles of the European Higher Education Area. This will aid in promoting the development of a high-quality medical teaching.
\end{abstract}

Keywords: Ebook, Medical Education, Surgery, Teaching Materials.

\section{INTRODUCCIÓN}

La Cirugía es la parte de la Medicina que tiene por objeto curar las enfermedades por medio de operaciones hechas con las manos o a través de instrumentos. Su nombre proviene del latín chirurgia, y éste del griego cheirourgia, de cheir: mano, y ergon: obra, trabajo. En el siglo XX, en paralelo al progreso científico, se desarrollaron las especializaciones en Medicina, unas de ellas más orientadas hacia el ejercicio clínico y otras, sin embargo, claramente orientadas al ámbito quirúrgico. Cabe reseñar, no obstante, que el clínico se ha hecho cada vez más intervencionista, y las sub-especializaciones de la cirugía hoy en día son, realmente, especialidades médico-quirúrgicas. Puesto que el hombre enferma como un todo, esta especialización en Medicina no puede entenderse como una mera compartimentalización. No se puede concebir la Cirugía independientemente de la Medicina. En consecuencia, la enseñanza de la Medicina no puede separarse de los principios que rigen la enseñanza de la Cirugía, y viceversa.

Hasta ahora, la enseñanza de la asignatura de "Procedimientos Quirúrgicos", que corresponde a los estudiantes de segundo curso del Grado en Medicina de la Universidad de Córdoba se ha llevado a cabo según el estilo tradicional; esto es, los estudiantes asisten a las clases teóricas que son impartidas por los diferentes profesores de la asignatura, los cuales explican los contenidos del temario con la ayuda de material gráfico tipo presentaciones en powerpoint, documentos pdf, imágenes y vídeos quirúrgicos. Los alumnos toman apuntes, completando así el contenido gráfico mostrado en clase con las explicaciones del profesor. Las dudas o preguntas se aclaran durante el transcurso de la clase o al finalizar la misma. En cuanto a la parte práctica de la asignatura, los alumnos realizan unas prácticas en el área quirúrgica del Hospital Universitario Reina Sofía, en las que asisten, en grupos de 2 alumnos, a las intervenciones quirúrgicas que se realizan en los quirófanos de Cirugía General y de Cirugía Torácica. A pesar de las ventajas del programa formativo, la reciente aparición de nuevas tecnologías pone al alcance del personal docente nuevas herramientas que podrían emplearse para optimizar la enseñanza y mejorar el rendimiento de los alumnos. Además, el carácter eminentemente práctico de la asignatura de Procedimientos Quirúrgicos hace necesario proporcionar a los alumnos abundante material videográfico para facilitar la comprensión y aprendizaje de los principios básicos de la Cirugía.

Se llevó a cabo un proyecto específico de elaboración de una herramienta multimedia, en concreto, un ebook, con el contenido teórico y práctico de la asignatura de Procedimientos Quirúrgicos, que se enmarca dentro de la Modalidad 1 del 
“Plan de Innovación y Buenas Prácticas Docentes 2016/2017”. Gracias a la preparación de este material docente, se pretendió mejorar la docencia médica según las directrices del Espacio Europeo de Educación Superior (EEES), fomentando así el desarrollo de una educación médica de calidad. Se seleccionó para este proyecto un material docente de gran utilidad para la enseñanza tanto del contenido teórico como del práctico: además de optimizar el tiempo de trabajo y el mejorar el rendimiento durante las clases, permitía su uso en ausencia del profesor e incluso fuera de la Institución, favoreciendo así la enseñanza virtual. Con la creación del EEES, se prima el desarrollo de habilidades y competencias y se valora el trabajo autónomo del alumno, lo cual implica necesariamente el uso de recursos didácticos potentes. El uso de ebook para este fin constituiría una metodología de gran valor para mejorar la formación universitaria, puesto que posibilitaría organizar y desarrollar actividades formativas en modalidad tanto presencial como no presencial. En este sentido, el uso del ebook, combinado con otras herramientas docentes tales como la plataforma e-learning de la Universidad de Córdoba, podría contribuir sin duda a potenciar la calidad de la docencia de la asignatura de Procedimientos Quirúrgicos, además de favorecer la comunicación e interacción entre profesores y alumnos. En última instancia, el uso de esta herramienta posibilitaría profundizar en el conocimiento y manejo de las tecnologías de la información y la comunicación (TICs) para innovar y mejorar la docencia en la educación médica.

\section{OBjeTIVOS}

Dado que en Cirugía gran parte del contenido temático consiste en el aprendizaje de técnicas (anudados, tipos de intervenciones, posiciones del paciente, etc), el uso de las nuevas tecnologías como el aprendizaje multimedia resulta esencial.

El principal objetivo del presente proyecto se basó en poner a disposición del alumno un libro en formato electrónico (ebook) con el contenido teórico y práctico de la asignatura de Procedimientos Quirúrgicos, que ayudase a potenciar la calidad de la docencia de la asignatura, mejorando el rendimiento del alumnado, favoreciendo el autoaprendizaje y reforzando el uso de las TICs en docencia médica. Los objetivos concretos del proyecto fueron los siguientes:

1. Elaboración de un libro electrónico (ebook) con el contenido teórico y práctico de la asignatura de Procedimientos Quirúrgicos.

2. Fomento de la utilización de los diferentes recursos didácticos (contenido teórico, material videográfico, casos prácticos, preguntas tipo test, etc.) para despertar y mantener el interés del alumnado y facilitar la comprensión de los contenidos de la asignatura de Procedimientos Quirúrgicos.

3. Facilitación a los alumnos el libre acceso a los recursos didácticos de la asignatura de Procedimientos Quirúrgicos presentados en forma de libro electrónico (contenido teórico, material videográfico, casos prácticos, preguntas tipo test, etc.) para fomentar el autoaprendizaje y la mejora individual de la formación en los principios quirúrgicos básicos.

\section{Metodología}

Para poder llevar a cabo los objetivos planteados en el proyecto, se desarrollaron una serie de actividades encaminadas a elaborar el contenido teórico correspondiente a cada tema de la asignatura, coleccionar un catálogo de imágenes quirúrgicas representativas, crear y editar material videográfico para su inclusión en el libro electrónico, crear una videoteca de técnicas quirúrgicas relacionadas con el contenido teórico de la asignatura, elaborar preguntas tipo test para reforzar el aprendizaje de los contenidos docentes y, finalmente, divulgar el material.

\section{- DESCRIPCIÓN}

1. Elaboración del contenido teórico correspondiente a cada tema de la asignatura: Para tal fin, se recopiló y analizó el temario de la asignatura facilitado clásicamente a los alumnos en forma de presentaciones en powerpoint. Se elaboró un índice para el libro electrónico en base al temario de la asignatura, al que se añadieron además otros apartados correspondientes a un catálogo de imágenes quirúrgicas, una videoteca y preguntas tipo test. Cada apartado teórico se desarrolló en forma de capítulo de libro electrónico, en el que se ha intercambiaron texto con imágenes y clips de vídeos, con objeto de facilitar el aprendizaje del alumno. El temario final del ebook consistió en los siguientes apartados:

a. Introducción a la cirugía: Referencias históricas. Derechos y deberes del cirujano. Derechos y deberes del paciente. Conceptos básicos (Estructura hospitalaria y quirófanos; personal necesario para la intervención; anestesia; analgesia; infección en cirugía; esterilidad, asepsia y antisepsia; respuesta biológica a la agresión; cicatrización y hemostasia.

b. Preoperatorio: Evaluación preoperatoria del paciente (evaluación preanestésica; evaluación quirúrgica; situación del enfermo en el preoperatorio inmediato). Información al enfermo (consentimiento informado; privacidad y confidencialidad; ventajas de la cirugía; riesgos de la cirugía; complicaciones del entorno quirúrgico). Instrumental quirúrgico (suturas; instrumental básico; instrumental avanzado). Indicaciones y contraindicaciones quirúrgicas (indicaciones de cirugía; contraindicaciones absolutas y relativas de la cirugía).

c. Intraoperatorio: Comportamiento del equipo quirúrgico en quirófano. Lavado de manos y colocación del equipo quirúrgico. Tipos de abordaje quirúrgico (cirugía abierta; cirugía endoscópica; cirugía radioguiada). Posiciones quirúrgicas 
(posiciones del paciente en quirófano; colocación del campo estéril). Incisiones (denominación de las incisiones quirúrgicas; ventajas e inconvenientes de cada incisión). Técnicas de anudado y sutura (tipos de suturas; tipos de agujas; técnicas de anudado; técnicas de sutura). Complicaciones y situaciones de riesgo intraoperatorio (control de la hemorragia; el hallazgo incidental; control y reparación de la yatrogenia; la muerte intraoperatoria).

d. Postoperatorio: Postoperatorio inmediato (recuperación post-anestésica; tratamiento post-quirúrgico; alta hospitalaria y revisión post-quirúrgica). Postoperatorio tardío (implicaciones sociales de la cirugía; consecuencias personales de una intervención quirúrgica; la complicación esperada; la complicación inesperada; de la recuperación ad integrum al exitus.

e. Catálogo de imágenes quirúrgicas: Se elaboró un catálogo de imágenes quirúrgicas representativas de los diferentes contenidos de la asignatura, para facilitar el aprendizaje del contenido teórico.

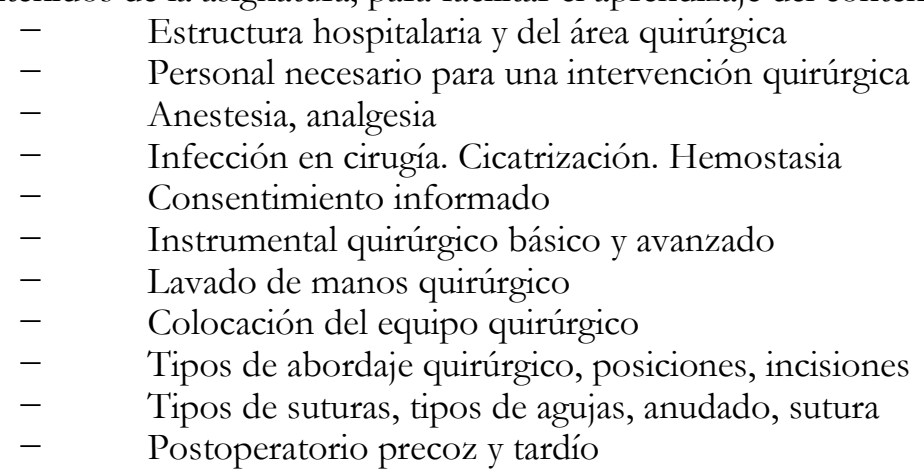

f. Videoteca: Se adquirieron vídeos correspondientes a los principales elementos teóricos de la asignatura. Se elaboró una colección de vídeos quirúrgicos, editados en formato AVI, MP4, y MPEG. A los clips de vídeos seleccionados, se le han incorporaron cuadros de texto o clips de voz con objeto de facilitar la comprensión del contenido, la asimilación de conocimientos, la relación de conceptos y la creación de nuevos interrogantes por parte del alumno. Tanto para la recopilación de imágenes como de vídeos quirúrgicos, se empleó un Ipad Pro adquirido específicamente para este proyecto, puesto que en el mismo dispositivo se reunían las propiedades de poseer cámara de fotos y video y, además, poder acceder al ebook de la asignatura.

g. Preguntas tipo test: se elaboraron preguntas tipo test basadas en el contenido teórico y práctico recogido en el ebook, con la finalidad de que el alumno asimilase los conocimientos adquiridos con cada tema, comprendiese el contenido presentado, y relacionase conceptos.

2. Elaboración de una colección de imágenes quirúrgicas relacionadas con los principios quirúrgicos básicos. Como ya se ha detallado en el apartado previo, se creó una colección de imágenes quirúrgicas representativas de los diferentes contenidos de la asignatura, que facilitasen el aprendizaje del contenido teórico. Se empleó un Ipad Pro adquirido específicamente para este proyecto. Las imágenes obtenidas fueron editadas y catalogadas para facilitar la asimilación de los contenidos de la asignatura.

3. Selección y edición del material videográfico para su inclusión en el libro electrónico. Del mismo modo que lo explicado para las imágenes quirúrgicas, se adquirieron vídeos quirúrgicos representativos de los diferentes elementos teóricos recogidos en el ebook. Dichos vídeos fueron editados para su posterior inclusión en el libro electrónico, insertando clips de audio o cuadros de texto que ayudasen al alumno a asimilar los conceptos.

4. Elaboración de una videoteca de técnicas quirúrgicas relacionadas con el contenido teórico de la asignatura. Los diferentes videos seleccionados, editados e incluidos en el libro electrónico representaban el principal contenido teórico de la asignatura, y su visualización por parte del alumno podría ayudar a mejorar su adquisición de competencias en la materia.

5. Elaboración de preguntas y actividades relacionadas con los diferentes contenidos de la asignatura.

Se creó un apartado de preguntas tipo test basadas en el contenido teórico y práctico recogido en el ebook. Con esto se pretendía facilitar la asimilación de conocimientos adquiridos con cada tema por parte del alumno, la comprensión del contenido presentado, y relacionar conceptos.

6. Divulgación del material docente.

Dada la experiencia positiva inicial obtenida en el grupo de trabajo, hemos decidido aplicar el proyecto docente en el curso 2017/2018, por lo que el libro electrónico de la asignatura de Procedimientos Quirúrgicos va a poder descargarse directamente del portal Web del Departamento de Especialidades Médico-Quirúrgicas, a través de la Plataforma Moodle de la Universidad de Córdoba [http://www3.uco.es/moodle/], accediendo a la asignatura de Procedimientos Quirúrgicos, para poder visualizarlo en cualquier dispositivo electrónico (ordenador o tableta) sin necesidad de conexión 
a internet. Así, el alumno va a poder acceder de manera individualizada o grupal a los distintos recursos didácticos de la asignatura, así como emplear las diferentes modalidades de enseñanza a distancia existentes en esta plataforma e-learning (presentaciones powerpoint, libro electrónico, catálogo de imágenes, videoteca, etc.) que facilitan el autoaprendizaje.

7. Autoevaluación de la experiencia.

Se llevó a cabo una valoración de la utilidad del proyecto de innovación docente mediante la distribución de encuestas de satisfacción en formato papel a un pequeño grupo de cinco alumnos y dos docentes de la asignatura de Procedimientos Quirúrgicos de la Facultad de Medicina de Córdoba al finalizar el curso académico 2016-2017. En dicha encuesta se formulaba una pregunta general: "Cuál es su nivel de satisfacción respecto a la aplicación del proyecto docente? Muy alto/Alto/Medio/Bajo/Muy Bajo”. Para ello, una vez finalizada la elaboración del ebook y terminado de impartirse el contenido teórico de la asignatura, se puso en marcha un grupo de trabajo con los referidos alumnos en una sesión de trabajo, en la cual se les mostró el libro electrónico en el Ipad Pro adquirido para tal fin. En dicha sesión de trabajo se les facilitó la familiarización al contenido del mismo. Finalizada la sesión de trabajo, se evaluó la experiencia mediante la distribución de una encuesta de satisfacción en formato papel, presentándose al finalizar la jornada los resultados de la misma, con objeto de poder determinar su utilidad como mejora de la calidad de la enseñanza de la asignatura.

\section{RESULTADOS OBTENIDOS}

Desde el punto de vista de la innovación docente, el principal resultado obtenido ha sido el desarrollo de competencias específicas en la aplicación del contenido teórico a la práctica, el uso de herramientas TICs, el fomento del empleo de diversos recursos didácticos (más allá de la mera "clase magistral"), así como el fomento del autoaprendizaje.

El alumno puede consultar el contenido del ebook en cualquier momento, ya sea en la propia Facultad o fuera de ella, visualizando aquellos subapartados que considere oportunos para completar y comprender el estudio teórico y práctico de la de la asignatura de Procedimientos Quirúrgicos.

Se recogieron 7 encuestas de satisfacción de la aplicación del proyecto docente en la jornada de trabajo específica. El porcentaje de alumnos y profesores que contestaron el cuestionario fue del $100 \%$. Como se muestra en la figura, el nivel de satisfacción en relación al proyecto docente fue, en general, muy elevado.

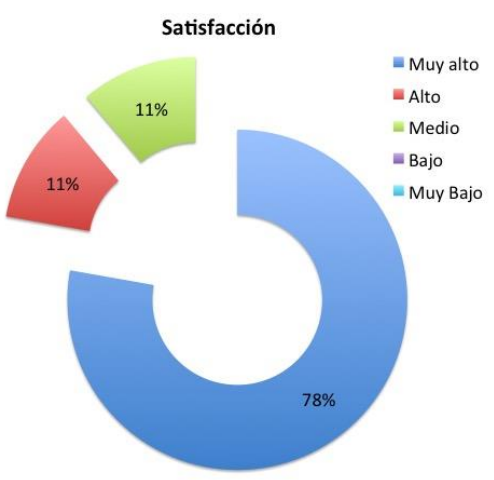

\section{UTILIDAD/ANÁLISIS}

Consideramos que la elaboración de docencia audiovisual en formato de libro electrónico relacionada con la asignatura de Procedimientos Quirúrgicos, empleando las TICs siguiendo un modelo de enseñanza e-learning, potencia el autoaprendizaje y la interactividad de los alumnos de la asignatura y, simultáneamente, permite desarrollar una metodología docente innovadora.

En nuestra opinión, este proyecto responde a las exigencias actuales del EEES y de la Universidad de Córdoba, puesto que contribuye a potenciar la calidad de la docencia en la asignatura de Procedimientos Quirúrgicos.

Consideramos de gran utilidad el presente proyecto de innovación docente, mediante el cual se recopiló en un mismo formato, el libro electrónico, el contenido teórico y práctico de la asignatura, cuyo aprendizaje se ve facilitado gracias a la incorporación de un catálogo de imágenes y vídeos quirúrgicos, así como numerosas preguntas tipo test. Este material ha sido de gran ayuda para mejorar la docencia presencial y no presencial en la asignatura de Procedimientos Quirúrgicos. En el mismo sentido, la difusión del ebook permitió al alumnado poder acceder a los contenidos del mismo mediante el uso de las TICs. Por otro lado, la elaboración de este proyecto permitió al profesorado de la asignatura ampliar y perfeccionar su formación en las metodologías de innovación de la calidad docente enfocadas a su aplicación en el EEES.

Mediante la elaboración de unas encuestas de evaluación al finalizar el curso académico, los alumnos han evaluado positivamente su utilidad para mejorar el aprendizaje de la asignatura. Dado que de estas encuestas se ha derivado una 
evaluación positiva, se ha planteado publicar el ebook para hacerlo disponible no ya a los usuarios de la Universidad de Córdoba, sino a toda la comunidad científica.

\section{CONCLUSIONES/DISCUSIÓN}

Consideramos que este proyecto de innovación docente nos ha permitido avanzar en el uso de nuevas tecnologías, como son las herramientas multimedia, para la docencia en el Grado en Medicina. En general y de la asignatura de Procedimientos Quirúrgicos, en particular. Con ello, creemos que se potencia la calidad de la docencia de dicha asignatura, puesto que el uso de esta herramienta facilita el poder profundizar en el conocimiento y manejo de las tecnologías de la información y la comunicación (TICs) para innovar y mejorar la docencia en la educación médica.

\section{BIBLIOGRAFÍA}

1. Agha RA, Papanikitas A, Baum M, Benjamin IS. The teaching of surgery in the undergraduate curriculum. Part II-Importance and recommendations for change. Int J Surg 2005;3(2):151-7.

2. Añel, E. (2008) Formación on-line en la Universidad. Pixel-Bit. Revista de Medios y Educación 2008;33:155-163.

3. Díaz-Barriga, F., Hernández, G (1999) Estrategias docentes para un aprendizaje significativo. Una interpretación constructivista. México: Mc. Graw Hill. ISBN 970-10-3526-7.

4. Fuller L, Lin G, Matsui JY, Sobotka SA, Cooke DT. Teaching surgery to medical students: perspectives from our mentees. Bull Am Coll Surg 2008;93(7):48-53.

5. Sweeney WB. Teaching surgery to medical students. Clin Colon Rectal Surg 2012;25(3):127-133. 